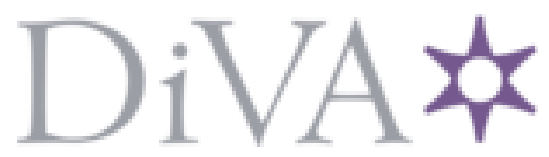

http://www.diva-portal.org

\title{
Postprint
}

This is the accepted version of a paper published in The American mathematical monthly. This paper has been peer-reviewed but does not include the final publisher proof-corrections or journal pagination.

Citation for the original published paper (version of record):

Eriksen, N. (2008)

The Freshman's Approach to Conway's Napkin Problem.

The American mathematical monthly, 115(6): 492-498

Access to the published version may require subscription.

N.B. When citing this work, cite the original published paper.

Permanent link to this version:

http://urn.kb.se/resolve?urn=urn:nbn:se:oru:diva-41705 


\title{
The freshman's approach to Conway's napkin problem
}

\author{
Niklas Eriksen
}

Arriving at a conference dinner and sitting down at any free space at a round table, one is usually confronted with a serious problem: "Should I pick the napkin on the right or on the left?" If this choice is made at random, some diners will probably have no napkin to choose from, both being taken by adjacent diners. What is the expected proportion of diners not getting a napkin?

This is the question posed by John Conway at a lunch at Bell Labs in 2001, as described in Peter Winkler's book Mathematical Puzzles: A Connoisseur's Collection [6]. To be more precise, we assume that all diners arrive one at a time and find seats at random (that is, independently and with uniform distribution over the remaining seats). A diner whose napkin par préférence is taken immediately has a go at the other napkin.

Winkler also gives the asymptotical solution, which is $(2-\sqrt{e})^{2} \approx 0.1234$. He provides two proofs, one with a combinatorial flavour and one probabilistic, originally given by Aidan Sudbury [4]. The latter one is more general in that it assigns the probability $p$ for prefering the right napkin and $q=1-p$ for prefering the left, arriving at $\left(1-p e^{q}\right)\left(1-q e^{p}\right) / p q$.

A more thorough analysis has recently been given by Claesson and Petersen [1], who use a system of equations containing seven generating functions to compute the generating function of the napkinless diners. This allows them to compute both the expectation and the variance of the number of napkinless diners as a function of the number $n$ of diners, as well as the corresponding figures for the number of diners who get a napkin, though not the one they wanted. These computations have been made in the general $p, q$ setting.

This increase in information is unfortunately also met by an increase in complexity of the solution. Defining these generating functions and discovering their relations, as well as introducing objects like ordered bipartitions, does not yield a one-line proof or even a one-page proof. However, the expected proportion of napkinless diners is very similar to its asymptotic, indicating that it can be solved in a simpler way, following Winkler and Sudbury.

This turns out to be quite true. We take the view of a freshman, knowing nothing of generating functions and other sophisticated methods, though being familiar with permutations and binomial cooefficients. Using only elementary facts about these objects, we compute the expected number of napkinless diners for table size $n$ and proportions $p$ and $q$ of diners preferring the right and left napkin, respectively. The approach is similar to that of Winkler [6] in his computation of the corresponding asymptotic, but more to the point.

We also use the same approach to give the first trivial computations of the expected number of frustrated diners. In addition, we solve a number of related problems, including that of the French diners and one concerning diners who use more than one napkin. 
$人$

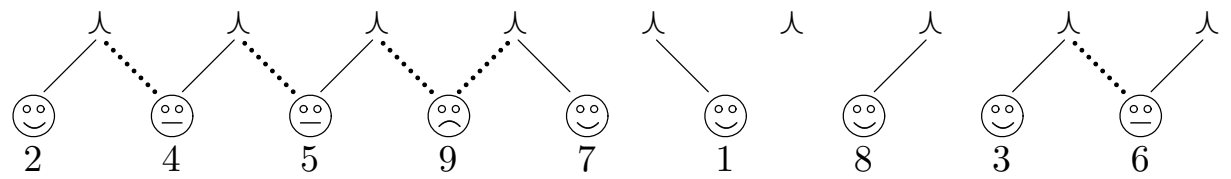

Figure 1: A part of a (round) table with diners and napkins. Solid sloped lines indicate that a diner takes a napkin and dotted lines that a diner attempts at, and fails in, taking a napkin. The numbers below indicate the order in which the diners arrive. The diners' smiles indicate whether they are happy, frustrated, or napkinless.

\section{The freshman's approach}

It is instructive to take a pictorial view of the setting, following Claesson and Petersen (see Figure 1). A solid line between a diner and one of the adjacent napkins indicates that the diner has taken this napkin, and a dotted line indicates that the diner has failed in taking that napkin. Thus, diners with a solid line only are happy, diners with both a solid and a dotted line are frustrated, and diners with two dotted lines do not get a napkin.

We will first compute the probability that diner $k$, that is the $k$ th diner to arrive, does not get a napkin. This can only happen if both the right and left napkins of diner $k$ have been taken. Thus, on each side of diner $k$ we have a number of diners arriving prior to diner $k$. To the right of $k$, there must be a closest diner having chosen the left napkin, and the diners in between have all tried to pick the right napkin, but failed and taken the left one instead. On the left side, the situation is mirrored. Such a segment is called a contraction (see Figure 2).

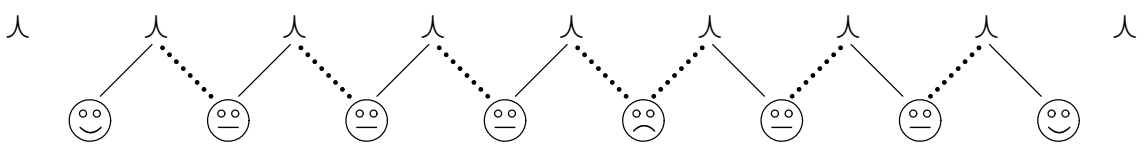

Figure 2: If a diner does not manage to take a napkin, then both adjacent napkins have already been taken. There is a closest happy diner on the left and on the right, and all diners between the closest happy diner and the napkinless diner must be frustrated. The segment from the left to the right closest happy diner is called a contraction.

Concentrating on a particular diner $k$, we may consider her position and choice of napkin to be set in advance. All other diners are positioned relative to her. We assume that the size of the contraction (number of diners) around diner $k$ is $m+1$ and that the number of diners on the left side in the contraction is $j$.

In total, the diners can position themselves in $(n-1)$ ! ways. However, to get a contraction the diners within the contraction must arrive in a certain order. The diners on the left side must arrive starting with the leftmost one, proceeding inwards, and similarly on the right side. Thus, the number of ways to position the diners to get a contraction is the number of possible ways to position those outside the contraction, $(n-m-1)$ !, times the number of ways to choose diners for the contraction, which is $\left(\begin{array}{c}k-1 \\ m\end{array}\right)\left(\begin{array}{c}m \\ j\end{array}\right)$. Also, while the diners outside the contraction may prefer any napkin, each diner within the contraction must 
prefer a specific napkin according to the definition of the contraction. For each diner in the contraction we therefore must multiply by the probability $1 / 2$ for the correct napkin preference.

We let $X_{n k}$ equal 1 if among $n$ diners, the $k$ th diner does not get a napkin, and 0 otherwise, and find that the probability of diner $k$ not getting a napkin is

$$
\mathbb{P}\left(X_{n k}=1\right)=\sum_{m=2}^{k-1} \sum_{j=1}^{m-1} \frac{\left(\begin{array}{c}
k-1 \\
m
\end{array}\right)\left(\begin{array}{c}
m \\
j
\end{array}\right)(n-m-1) !}{(n-1) !}\left(\frac{1}{2}\right)^{m} .
$$

Generalising to the probabilities $p$ and $q=1-p$ for prefering the right and left napkin, we get

$$
\begin{aligned}
\mathbb{P}\left(X_{n k}=1\right) & =\sum_{m=2}^{k-1} \sum_{j=1}^{m-1}\left(\begin{array}{c}
k-1 \\
m
\end{array}\right)\left(\begin{array}{c}
m \\
j
\end{array}\right) \frac{(n-m-1) !}{(n-1) !} q^{j} p^{m-j} \\
& =\sum_{m=2}^{k-1}\left(\begin{array}{c}
k-1 \\
m
\end{array}\right) \frac{(n-m-1) !}{(n-1) !} p^{m} \sum_{j=1}^{m-1}\left(\begin{array}{c}
m \\
j
\end{array}\right)\left(\frac{q}{p}\right)^{j} \\
& =\sum_{m=2}^{k-1}\left(\begin{array}{c}
k-1 \\
m
\end{array}\right) \frac{(n-m-1) !}{(n-1) !} p^{m}\left(\left(1+\frac{q}{p}\right)^{m}-1-\left(\frac{q}{p}\right)^{m}\right) \\
& =\sum_{m=2}^{k-1}\left(\begin{array}{c}
k-1 \\
m
\end{array}\right) \frac{(n-m-1) !}{(n-1) !}\left(1-\left(p^{m}+q^{m}\right)\right) .
\end{aligned}
$$

Note that we use nothing more than the binomial theorem and the fact that $p+q=1$ to obtain this formula. We note that the probability that the last diner does not get a napkin converges to such a low value as

$$
\begin{aligned}
& \sum_{m=2}^{n-1}\left(\begin{array}{c}
n-1 \\
m
\end{array}\right) \frac{(n-m-1) !}{(n-1) !}\left(1-\left(p^{m}+q^{m}\right)\right) \\
= & \sum_{m=2}^{n-1} \frac{1-\left(p^{m}+q^{m}\right)}{m !} \\
= & \exp _{n-1}(1)-2-\left(\exp _{n-1}(p)-1-p+\exp _{n-1}(q)-1-q\right) \\
= & \left(1-\exp _{n-1}(p)\right)\left(1-\exp _{n-1}(q)\right) .
\end{aligned}
$$

Above, $\exp _{n}(x)$ refers to the truncated exponential function $\sum_{k=0}^{n} x^{k} / k$ !. In particular, for $p=q=1 / 2$ we get asymptotically $\left(e^{1 / 2}-1\right)^{2} \approx 0.4208$.

Summing over all diners, we compute the expected number of napkinless diners, $X_{n}=$ 
$\sum X_{n k}$, as

$$
\begin{aligned}
\mathbb{E}\left(X_{n}\right) & =\sum_{k=1}^{n} \sum_{m=2}^{k-1}\left(\begin{array}{c}
k-1 \\
m
\end{array}\right) \frac{(n-m-1) !}{(n-1) !}\left(1-\left(p^{m}+q^{m}\right)\right) \\
& =\sum_{m=2}^{n-1} \frac{(n-m-1) !}{(n-1) !}\left(1-\left(p^{m}+q^{m}\right)\right) \sum_{k=m+1}^{n}\left(\begin{array}{c}
k-1 \\
m
\end{array}\right) \\
& =\sum_{m=2}^{n-1} \frac{(n-m-1) !}{(n-1) !}\left(1-\left(p^{m}+q^{m}\right)\right)\left(\begin{array}{c}
n \\
m+1
\end{array}\right) \\
& =n \sum_{m=2}^{n-1} \frac{1-\left(p^{m}+q^{m}\right)}{(m+1) !} \\
& =n\left(\left(\exp _{n}(1)-\frac{5}{2}\right)-\frac{1}{p}\left(\exp _{n}(p)-1-p-\frac{p^{2}}{2}\right)-\frac{1}{q}\left(\exp _{n}(q)-1-q-\frac{q^{2}}{2}\right)\right) \\
& =n \frac{\left(1-p \exp _{n}(q)\right)\left(1-q \exp _{n}(p)\right)}{p q} .
\end{aligned}
$$

The result agrees with the $p q$ asymptotics given by Sudbury [4].

\section{Generalisations and related problems}

\subsection{Frustrated diners}

The freshman's approach lends itself to many similar problems. For instance, Claesson and Petersen considered the number of frustrated diners as well, those being the ones that got a napkin, although not the one they wanted. Let the indicative function of the frustrated diners be $Y_{n k}$. By considering right and left halves of contractions, we find the probability that diner $k$ does not get her favourite napkin (and possibly none at all) to be

$$
\mathbb{P}\left(X_{n k}+Y_{n k}=1\right)=\sum_{m=1}^{k-1}\left(\begin{array}{c}
k-1 \\
m
\end{array}\right) \frac{(n-m-1) !}{(n-1) !}\left(q p^{m}+p q^{m}\right) .
$$

Summing this over all $k$ gives, with $Y_{n}=\sum Y_{n k}$,

$$
\begin{aligned}
\mathbb{E}\left(X_{n}+Y_{n}\right) & =n \sum_{m=1}^{n-1} \frac{q p^{m}+p q^{m}}{(m+1) !} \\
& =n\left(\frac{q}{p}\left(\exp _{n}(p)-1-p\right)+\frac{p}{q}\left(\exp _{n}(q)-1-q\right)\right) .
\end{aligned}
$$

While it seems hard to make a really nice expression of this, we can count the diners who do not get a napkin at all twice, to obtain the number of failed attempts to grab a napkin. We get

$$
\mathbb{E}\left(X_{n}+Y_{n}\right)+\mathbb{E}\left(X_{n}\right)=n\left(1-\exp _{n}(p)\right)\left(1-\exp _{n}(q)\right)
$$


Of course, if we instead remove the number of diners who do not get a napkin, we obtain the number of frustrated diners:

$$
\mathbb{E}\left(Y_{n}\right)=\frac{n}{p q}\left(\left(p+p \exp _{n}(q)\right)\left(q+q \exp _{n}(p)\right)-2\left(1-p \exp _{n}(q)\right)\left(1-q \exp _{n}(p)\right)\right) .
$$

This confirms the asymptotic for the number of frustrated diners for $p=q=1 / 2$ obtained in $[1]$.

\subsection{Happy diners}

It would be interesting to compute the probability that exactly $k$ diners are unable to get a napkin. Unfortunately, this seems quite hard. However, as shown by Claesson and Peterson, there is a special case that presents few obstacles, namely $k=0$. What is the probability that all diners get a napkin?

Assume, without loss of generality, that the first diner chooses the right napkin, and number the seats starting with the seat to the right of the first diner, progressing to the right. Let $\pi_{i}$ equal $k-1$ if the $k$ th diner sits in seat number $i$. Then $\pi$ is a permutation on $\{1, \ldots, n-1\}$. Assuming that all diners have a napkin, they must also have taken the one on their right side. Diners arriving after their left neighbour will be forced to take the right napkin regardles of which they prefer, but diners arriving before their left neighbour must prefer the right napkin. Arriving before the left neighbour corresponds to a descent in the permutation $\pi$. The number of permutations of $n-1$ elements with $k-1$ descents is given by the Eulerian numbers $A(n-1, k)$ and the sums $A_{n}(x)=\sum A(n, k) x^{k}$ are the Eulerian polynomials (see for instance [3]).

There are $A(n-1, k) /(n-1)$ ! permutations with $k-1$ descents, and the probability that each diner following a descent prefers the right napkin is $(1 / 2)^{k-1}$. We thus want to compute

$$
\sum_{k=1}^{n-1} \frac{A(n-1, k)}{(n-1) !}\left(\frac{1}{2}\right)^{k-1}=\frac{2 A_{n-1}(1 / 2)}{(n-1) !} .
$$

Plugging $u=2^{-1}$ into

$$
\sum_{k \geq 0} k^{n} u^{k}=\frac{A_{n}(u)}{(1-u)^{n+1}}
$$

(taken from [2]), we arrive at $2^{n+1} A_{n}(1 / 2)=\sum k^{n} / 2^{k}$ with asymptotic $n ! / \log ^{n+1}(2)$ [5]. Thus, the probability becomes

$$
\frac{\sum_{k \geq 0} \frac{k^{n-1}}{2^{k}}}{(n-1) ! 2^{n-1}} \approx \frac{2}{(2 \log (2))^{n}} .
$$

\subsection{French diners}

According to Claesson and Petersen, French combinatorialist Sylvie Corteel remarked that a French diner would rather refuse to eat than take the undesired napkin. This leads them to mention the problem of how the number of napkinless diners changes with the proportion of French diners, who do not take a napkin unless it is the preferred one. Using 
the techniques above, this is not hard to compute. While it can be done for any probability $p$ of right-oriented diners, we restrict ourselves to $p=1 / 2$ for clarity.

Let $f$ be the probability that a diner is French. If diner $k$ is not French, then both adjacent napkins must be taken for the diner to be without. Also, only the two outermost diners in the contraction can be French. Thus, the probability that $k$ is not French and without napkin is

$$
(1-f) \sum_{m=2}^{k-1} \sum_{j=1}^{m-1}\left(\begin{array}{c}
k-1 \\
m
\end{array}\right)\left(\begin{array}{c}
m \\
j
\end{array}\right) \frac{(n-m-1) !}{(n-1) !}\left(\frac{1}{2}\right)^{m}(1-f)^{m-2},
$$

which sums over $k$ to

$$
\frac{n}{(1-f)^{2}}\left(\left(\exp _{n}\left(\frac{1-f}{2}\right)-2\right)^{2}-f\right)
$$

On the other hand, French diners need only to be frustrated to be without a napkin. The probability that this happens is

$$
f \sum_{m=1}^{k-1}\left(\begin{array}{c}
k-1 \\
m
\end{array}\right) \frac{(n-m-1) !}{(n-1) !}\left(\frac{1}{2}\right)^{m}(1-f)^{m-1},
$$

and summing over $k$ gives

$$
\frac{n f}{(1-f)^{2}}\left(2 \exp _{n}\left(\frac{1-f}{2}\right)-3+f\right) .
$$

Adding these two alternatives gives the expected proportion of napkinless diners for any $f$, which becomes

$$
\left(1-\frac{\exp _{n}\left(\frac{1-f}{2}\right)-1}{1-f}\right)^{2}
$$

If we let $f$ increase from 0 to 1 , this is an almost linear function starting at $0.1234 \ldots$ and ending at $1 / 4$.

\subsection{Dinner conversations on mathematics}

In an informal survey conducted during the preparation of [1] on which napkin they preferred, one person answered "I would use the napkin on my left to wipe my mouth, and the one on my right to write on." To be able to discuss mathematics during the meal, the diner takes both napkins, if they are free.

Imagine that the dinner party consists of four kinds of diners: those who prefer the left napkin, those who prefer the right, those who try to take both napkins, and those who use the table cloth for wiping their mouths and writing on, not taking any napkin even if it is available. This extends the possible levels of frustration well beyond what we can keep record of, so let's concentrate on the napkins instead of the diners. What proportion of napkins is expected to be unused? Let $p_{r}, p_{\ell}, p_{b}$, and $p_{n}$ denote the probabilities that the diner tries to take the right, the left, both, and none of the napkins, respectively. 
We will first compute the probability that diner $k$ tries to take the napkin to her left, and then reduce this number by the probability that she fails. The $k$ th diner tries to take the left napkin either by choice or being forced to by her right neighbours. In any case, there is a half contraction in which the rightmost diner prefers the left or both napkins, and in between we have some number of diners who are forced to take the left napkin, though preferring the right. Thus, the probability that diner $k$ attempts for the left napkin is

$$
\sum_{m=0}^{k-1}\left(\begin{array}{c}
k-1 \\
m
\end{array}\right) \frac{(n-m-1) !}{(n-1) !} p_{r}^{m}\left(p_{\ell}+p_{b}\right)
$$

which sums over $k$ to

$$
n \frac{p_{\ell}+p_{b}}{p_{r}}\left(\exp _{n}\left(p_{r}\right)-1\right) .
$$

On the other hand, the probability that diner $k$ attempts for the left napkin and fails is, by a similar argument with full contraction,

$$
\begin{aligned}
& \sum_{m=1}^{k-1} \sum_{j=1}^{m}\left(\begin{array}{c}
k-1 \\
m
\end{array}\right)\left(\begin{array}{c}
m \\
j
\end{array}\right) \frac{(n-m-1) !}{(n-1) !} p_{r}^{m-j} p_{\ell}^{j-1}\left(p_{r}+p_{b}\right)\left(p_{\ell}+p_{b}\right) \\
& =\frac{\left(p_{r}+p_{b}\right)\left(p_{\ell}+p_{b}\right)}{p_{\ell}} \sum_{m=1}^{k-1}\left(\begin{array}{c}
k-1 \\
m
\end{array}\right) \frac{(n-m-1) !}{(n-1) !}\left(\left(p_{r}+p_{\ell}\right)^{m}-p_{r}^{m}\right),
\end{aligned}
$$

which sums over $k$ to

$$
n \frac{\left(p_{r}+p_{b}\right)\left(p_{\ell}+p_{b}\right)}{p_{r} p_{\ell}\left(p_{r}+p_{\ell}\right)}\left(p_{r} \exp _{n}\left(p_{r}+p_{\ell}\right)-\left(p_{r}+p_{\ell}\right) \exp _{n}\left(p_{r}\right)+p_{\ell}\right) .
$$

Subtracting (2) from (1) gives the expected number of napkins taken from the right. We add to this the corresponding expectation of napkins taken from the left and remove it all from $n$ to obtain the expected number of remaining napkins. It is

$$
\begin{aligned}
n- & \left(n \frac{p_{\ell}+p_{b}}{p_{r}}\left(\exp _{n}\left(p_{r}\right)-1\right)+n \frac{p_{r}+p_{b}}{p_{\ell}}\left(\exp _{n}\left(p_{\ell}\right)-1\right)\right. \\
& \left.-n \frac{\left(p_{r}+p_{b}\right)\left(p_{\ell}+p_{b}\right)}{p_{r} p_{\ell}}\left(\exp _{n}\left(p_{r}+p_{\ell}\right)-\exp _{n}\left(p_{r}\right)-\exp _{n}\left(p_{\ell}\right)+1\right)\right) \\
= & n\left(1-\frac{p_{\ell}+p_{b}}{p_{r}}\left(\exp _{n}\left(p_{r}\right)-1\right)\right)\left(1-\frac{p_{r}+p_{b}}{p_{\ell}}\left(\exp _{n}\left(p_{\ell}\right)-1\right)\right) .
\end{aligned}
$$

The special case $p_{r}=p_{\ell}=p_{b}=p_{n}=1 / 4$ thus gives the proportion $\left(3-2 e^{1 / 4}\right)^{2} \approx 0.1866$ of unused napkins, which is about 50 percent higher than 0.1234 from the original setting.

\section{Acknowledgments}

I am endebted to Anders Claesson for introducing me to this problem in a first class seminar, and for sharing his ideas with me during the preparation of this manuscript. 


\section{References}

[1] A. Claesson and K. Petersen, Conway's napkin problem, this Monthly 114 (2007) $217-231$.

[2] L. Comtet, Advanced Combinatorics, D. Reidel, Dordrecht, The Netherlands, 1974.

[3] R. Stanley, Enumerative Combinatorics, vol. 1, Cambridge University Press, Cambridge, 1997.

[4] A. Sudbury, Inclusion-exclusion methods for treating annihilating and deposition processes, J. Appl. Probab. 39 (2002) 466-478.

[5] H. Wilf, generatingfunctionology, Academic Press, New York, NY, 1990.

[6] P. Winkler, Mathematical Puzzles: A Connoisseur's Collection, A K Peters, Natick, MA, 2004.

Niklas Eriksen received his M.Sc. from the Royal Institute of Technology in Stockholm, Sweden, in 1999 and his Ph.D. in 2003 from the same university. He is currently an assistant professor at Göteborg University, where he continues his work on combinatorics in general and combinatorial aspects of comparative genomics in particular.

Department of Mathematical Sciences, Göteborg University and Chalmers University of Technology, SE-412 96 Göteborg, Sweden

ner@math.chalmers.se 